\title{
Retrospective Analysis of Training Intensity Distribution Based on Race Pace Versus Physiological Benchmarks in Highly Trained Sprint Kayakers
}

\author{
Manuel Matzka', Robert Leppich², Billy Sperlich ${ }^{1 *}$ (D) and Christoph Zinner ${ }^{3}$
}

\begin{abstract}
Background: Research results on the training intensity distribution (TID) in endurance athletes are equivocal. This non-uniformity appears to be partially founded in the different quantification methods that are implemented. So far, TID research has solely focused on sports involving the lower-body muscles as prime movers (e.g. running). Sprint kayaking imposes high demands on the upper-body endurance capacity of the athlete. As there are structural and physiological differences between upper- and lower-body musculature, TID in kayaking should be different to lowerbody dominant sports. Therefore, we aimed to compare the training intensity distribution during an 8-wk macrocycle in a group of highly trained sprint kayakers employing three different methods of training intensity quantification.
\end{abstract}

Methods: Heart rate (HR) and velocity during on-water training of nine highly trained German sprint kayakers were recorded during the final 8 weeks of a competition period leading to the national championships. The fractional analysis of TID was based on three zones ( $Z$ ) derived from either $\mathrm{HR}\left(\mathrm{TID}_{\mathrm{Bla}-\mathrm{HR}}\right)$ or velocity $\left(\mathrm{TID}_{\mathrm{Bla-}-\mathrm{V}}\right)$ based on blood lactate $\left(B_{l a}\right)$ concentrations $\left(Z 1 \leq 2.5 \mathrm{mmol} \mathrm{L}^{-1} \mathrm{~B}_{\mathrm{la}}, \mathrm{Z} 2=2.5-4.0 \mathrm{mmol}^{-1} \mathrm{~B}_{\mathrm{la}}, Z 3 \geq 4.0 \mathrm{mmol} \mathrm{L}^{-1} \mathrm{~B}_{\mathrm{la}}\right.$ ) of an incremental test or the $1000-\mathrm{m}$ race pace $\left(\mathrm{TID}_{\text {Race }}\right): \mathrm{Z1} \leq 85 \%$ of race pace, $Z 2=86-95 \%$ and $Z 3 \geq 95 \%$.

Results: $\operatorname{TID}_{\text {Bla-V }}(Z 1: 68 \%, Z 2: 14 \%, Z 3: 18 \%)$ differed from $\operatorname{TID}_{\text {Bla-HR }}(Z 1: 91 \%, Z 2: 6 \%, Z 3: 3 \%)$ in each zone (all $\left.p<0.01\right)$. $\operatorname{TID}_{\text {Race }}(Z 1: 73 \%, Z 2: 20 \%, Z 3: 7 \%)$ differed to $Z 3$ in $\operatorname{TID}_{\text {Bla-v }}(p<0.01)$ and all three $\mathrm{TID}_{\text {Bla-HR }}$ Zones (all $\left.p<0.01\right)$. Individual analysis revealed ranges of $Z 1, Z 2, Z 3$ fractions for $T_{I I} D_{\text {Bla-HR }}$ of $85-98 \%, 2-11 \%$ and $0.1-6 \%$. For $T_{I D} D_{B l a-v}$, the individual ranges were $41-82 \%(Z 1), 6-30 \%$ (Z2) and 8-30\% (Z3) and for TID Race $64-81 \%(Z 1), 14-29 \%(Z 2)$ and 4-10\% (Z3).

Conclusion: The results show that the method of training intensity quantification substantially affects the fraction of $T I D$ in well-trained sprint kayakers. $T I D_{\text {Race }}$ determination shows low interindividual variation compared to the physiologically based $T_{I D} D_{\text {Bla-HR }}$ and $T I D_{\text {Bla-v }}$. Depending on the aim of the analysis $T I D_{\text {Race, }} T D_{\text {Bla-HR }}$ and $T_{I D}$ Bla-v have advantages as well as drawbacks and may be implemented in conjunction to maximize adaptation.

Keywords: Endurance training, Sprint kayaking, Training zones, Polarized training, Pyramidal training, Upper-body sport

\section{Key Points}

*Correspondence: billy.sperlich@uni-wuerzburg.de

${ }^{1}$ Integrative and Experimental Exercise Science and Training, University

of Würzburg, Würzburg, Germany

Full list of author information is available at the end of the article
- TID $_{\text {Bla-HR }}$ reveals a pyramidal TID for Z1, Z2 and Z3 (91 $\pm 4 \%, 6 \pm 2 \%, 3 \pm 2 \%)$, whereas $\mathrm{TID}_{\text {Bla-V }}$ showed a TID with a tendency towards a polarized TID ( $68 \pm 14 \%, 14 \pm 8 \%$ and $18 \pm 8 \%)$. 
- Based on the present data, a combination of both TID $_{\text {Bla-HR }}$ and TID Bla-V may yield value for TID quantification in kayak sprinting with TID $_{\text {Bla-HR }}$ implemented to determine $\mathrm{Z} 1$ and $\mathrm{Z} 2$ and $\mathrm{TID}_{\mathrm{Bla}-\mathrm{V}}$ for $\mathrm{Z} 3$.

- The analysis of TID $_{\text {Race }}$ showed a pyramidal TID pattern (Z1: $73 \pm 5 \%, Z 2: 20 \pm 5 \%, Z 3: 7 \pm 2 \%)$ on the group level.

- The most noticeable difference between the TID determination methods is the comparably low interindividual variation in TID for TID $_{\text {Race }}$.

\section{Introduction}

Endurance athletes structure their training intensity usually based on different intensity zone models [1-4]. Most commonly, a three-zone model is implemented to distinguish the distribution of training between low, moderate and high intensities. The zones are generally determined based on physiological parameters (e.g. maximum oxygen uptake $\left[\mathrm{VO}_{2 \max }\right]$, blood lactate $\left.\left[\mathrm{B}_{\mathrm{la}}\right]\right)$ established from incremental testing. Zone $(\mathrm{Z}) 1$ defines intensities below the aerobic threshold $\left(<2.5 \mathrm{mmol} \mathrm{L}^{-1} \mathrm{~B}_{\mathrm{la}}\right.$ or $\leq 80 \%$ of $\left.\mathrm{VO}_{2 \max }\right), \mathrm{Z} 2$ relates to intensities between aerobic and anaerobic threshold $\left(2.5-4.0 \mathrm{mmol} \mathrm{L} \mathrm{L}^{-1} \mathrm{~B}_{\mathrm{la}}\right.$ or $81-87 \%$ of $\left.\mathrm{VO}_{2 \max }\right)$ and $\mathrm{Z3}$ equals intensities above the anaerobic threshold ( $>4.0 \mathrm{mmol} \mathrm{L}^{-1} \mathrm{~B}_{\mathrm{la}}$ or $\geq 88 \%$ of $\mathrm{VO}_{2 \max }$ ) [5]. Different internal (e.g. heart rate [HR], $\mathrm{VO}_{2}$, blood lactate) and/or external (e.g. velocity, power output) parameters are continuously monitored to quantify these intensity zones during daily training.

Besides the consensus in research regarding the physiologically based zone demarcation, different TIDs have been found in endurance athletes. Currently, the debate about the superiority of a distinct TID model appears to focus on two models that were mostly found to be implemented by elite endurance athletes [4]: (i) The polarized TID consists of high volumes in Z1 and Z3, with only small proportions in $\mathrm{Z} 2$. Proportion of $\mathrm{Z1}$ is generally higher than $\mathrm{Z} 3$ and proportion of $\mathrm{Z3}$ is always higher than Z2. (ii) The pyramidal TID is characterized by high volumes of $\mathrm{Z} 1$ and gradually decreasing percentages in $\mathrm{Z} 2$ and $\mathrm{Z} 3$.

To date, research fails to give a clear answer, as to whether one of these TID models is superior. This nonuniformity appears to be partially founded in the different quantification methods that are implemented [6]. For example, a comparison of heart rate and velocity-based measures of TID in elite middle- and long-distance runners during an 8-week general preparation phase found a polarized TID when training was quantified by running speed (Z1: $79.9 \% \pm 7.3 \%, \quad Z 2: 5.3 \% \pm 4.9 \%, \quad Z 3$ : 14.7\% $\pm 7.3 \%$; Polarization-Index [Pol-Index]: 2.3 a.u.) and a pyramidal structured TID (Z1: $79.6 \% \pm 7.2 \%, \mathrm{Z2}$ :
17.0\% $\pm 6.3 \%$, Z3: $3.4 \% \pm 2.0 \%$; Pol-Index: 1.2 a.u.) when the training zones where quantified on HR [7].

Kenneally et al. recently created a novel way to determine endurance intensity zones in middle- and longdistance running based on the relative intensity of the athletes' competition performance (percentage of race pace) $[7,8]$. This new method is based on the fact, that previous research on the effects of different endurance training TID mainly analysed changes in physiological variables or time trial performances rather than actual competition performance, as the main aim of training. However, competition performance is influenced by multiple internal (e.g. central nervous system, biomechanics, cardiopulmonary system, etc.) and external factors (environment, tactics, etc.) and cannot be solely described by physiological variables [7]. In order to develop certain physiological systems (e.g. oxygen uptake, lactate threshold, exercise economy) it is well known that time spent in certain physiologically based intensity zones induce specific adaptation related to each zone [9]. Nevertheless, physiologically based intensity zones may be of limited value especially when training periods aim to specifically fine-tune the development of competition performance. Therefore, the race pace the race pace may serve as a better reference point to prescribe and analyse training intensity. The race pace-based approach aims to take different performance determinants of the competition performance into account [7], however this approach has its flaws as well. For example the TID of middle- and longdistance runners show markedly less interindividual differences when analysed based on race pace compared to TID analysis based on physiological benchmarks [7].

In contrast to endurance running, sprint kayaking imposes high demands on the upper-body endurance capacity of the athlete, as the athlete propels the boatbody-system against water resistance involving his/her relatively small upper-body muscles [10]. Differences in muscle mass, muscle fibre spectrum, oxygen extraction [11] as well as glucose and lipid oxidative capacity [11-13] between upper and lower body require different qualitative and quantitative training stimuli for distinct adaptation. From this perspective, the TID in kayak sprinting when compared to other leg-dominated sports should be different. Additionally, opposed to Olympic running-events with relatively high similarity of the courses, sports like kayak sprinting that are far more influenced by course characteristics (e.g. lake vs. artificial regatta course) and environmental factors (e.g. waves and water temperature) may not have the same potential to incorporate race pace-based velocity measures as a valuable tool for TID analysis [8].

So far, no study compared an internal and an external parameter-based method for determining TID over the 
course of a training cycle in kayak sprinting. Furthermore, no investigation implemented a race pace-based approach for TID analysis. Therefore, the current study aimed to retrospectively compare different TID quantification methods based on physiologically determined intensity zones using heart rate and velocity monitoring as well as race pace-based zone determination using velocity monitoring during an 8-week competition period in highly trained kayak sprint athletes.

\section{Materials and Methods}

\section{Experimental Design}

This retrospective observational study was part of a 1 -year study-project and took place during the season 2020 . The present data were collected from June until August 2020. The observation comprised the final 8 weeks of the competition period before the German national championships which took place the week after data collection ended. Figure 1 illustrates the time course, methods involved and parameters obtained during the study period.

\section{Participants}

The nine athletes were recruited from two different official performance headquarters of the German and/or North Rhine-Westphalian Canoe Federation. The key anthropometric, physiological and performance characteristics of the flatwater sprint kayakers are summarized in Table 1.

Four of the nine participants were members of the German Development Team and five were part of the Western German Regional Team. All participants competed on the highest national level in Germany. The athletes competed in kayaking for at least 6 years.

All were familiar with all testing procedures employed, having experienced frequent testing throughout their career. All procedures were approved by the institute's ethics committee and conducted in accordance with the Declaration of Helsinki. After being informed in detail about the risks, benefits and procedures, all participants and their legal guardians gave their written consent to participate in this study.

\section{Determination of Intensity Zones}

The HR (TID Bla-HR $)$ and velocity $\left(\mathrm{TID}_{\text {Bla-V }}\right)$ zones were based on the HR and velocity corresponding to certain blood lactate levels obtained from the incremental step test. The zones for $\mathrm{TID}_{\mathrm{Bla}-\mathrm{HR}}$ and $\mathrm{TID}_{\mathrm{Bla}-\mathrm{V}}$ were established based on the results of an incremental step test performed before the 8-week macrocycle. HR and velocity for each zone were based on previous categorization [5] with Z1 as $\mathrm{HR}$ and velocity corresponding to $>60 \%$

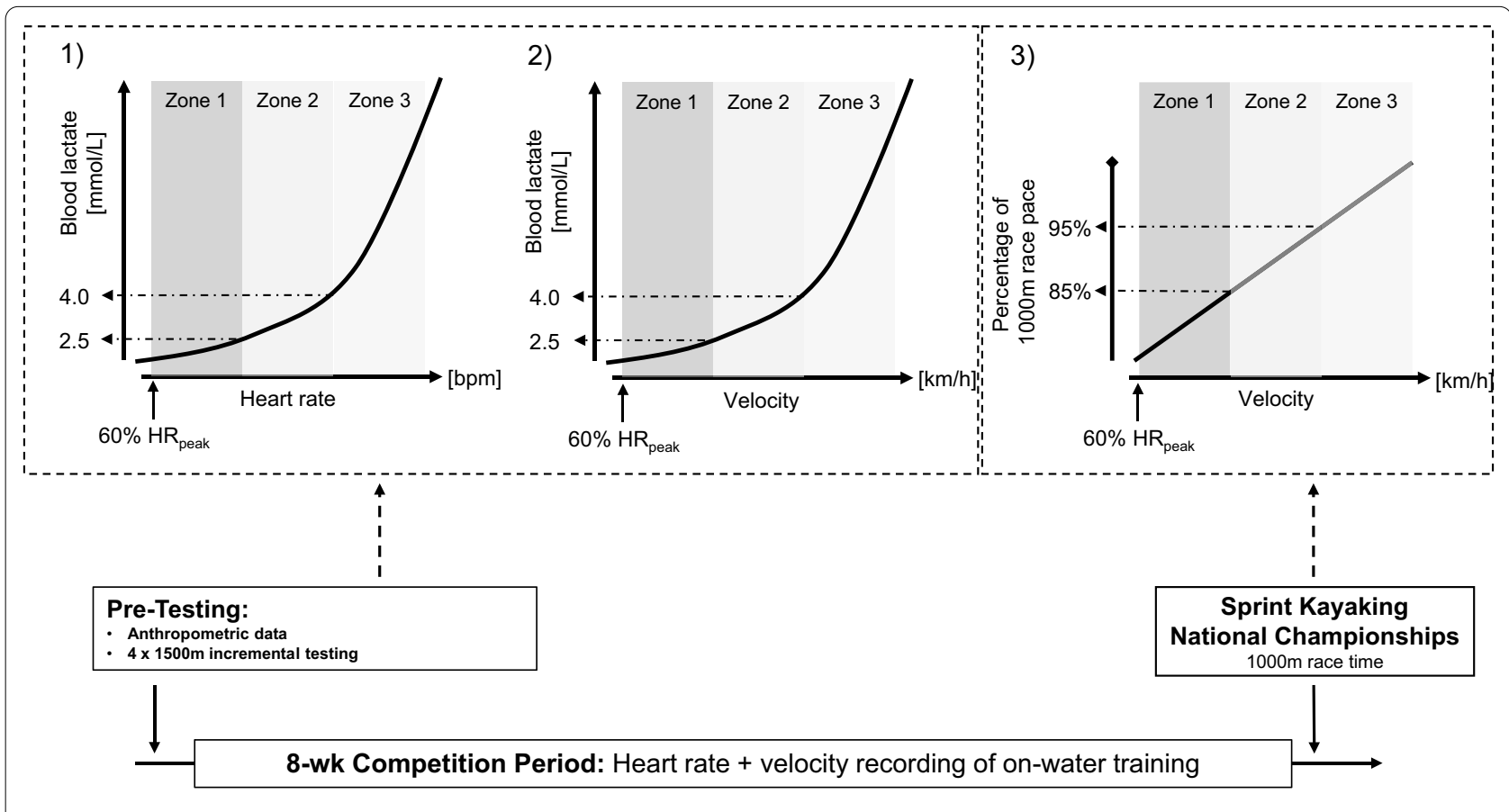

$\mathrm{HR}_{\text {peak }}=$ peak heart rate

Fig. 1 The overall study design with the three different methods of TID quantification. (1) Heart rate-based zones from blood lactate analysis during incremental testing. (2) Velocity-based zones from blood lactate analysis during incremental testing. (3) Velocity-based zones from $1000 \mathrm{~m}$ race time 
Table 1 Participants' characteristics

\begin{tabular}{|c|c|c|c|c|c|c|c|c|c|}
\hline \multirow[t]{2}{*}{ P-ID } & \multirow[t]{2}{*}{ Sex } & \multirow[t]{2}{*}{$\begin{array}{l}\text { Age } \\
\text { (years) }\end{array}$} & \multirow[t]{2}{*}{$\begin{array}{l}\text { Height } \\
\text { (cm) }\end{array}$} & \multirow[t]{2}{*}{$\begin{array}{l}\text { Body Mass } \\
(\mathrm{kg})\end{array}$} & \multirow{2}{*}{$\begin{array}{l}\text { Body Mass } \\
\text { Index } \\
\left(\mathrm{kg} / \mathrm{m}^{2}\right)\end{array}$} & \multirow{2}{*}{$\begin{array}{l}\text { Peak oxygen } \\
\text { uptake } \\
\text { (ml/min) }\end{array}$} & \multicolumn{3}{|c|}{$\begin{array}{l}\text { Best times at Sprint Kayaking } \\
\text { National Championships (s) }\end{array}$} \\
\hline & & & & & & & $200 \mathrm{~m}$ & $500 \mathrm{~m}$ & $1000 \mathrm{~m}$ \\
\hline 1 & Male & 17 & 182 & 82.5 & 24.9 & 5275 & $<38.3$ & $<105.1$ & $<224.1$ \\
\hline 2 & Male & 18 & 195 & 87.3 & 23.0 & 5347 & $<40.1$ & $<105.6$ & $<225.6$ \\
\hline 3 & Male & 23 & 179 & 73.8 & 23.0 & 5209 & n.d & $<105.8$ & $<222.2$ \\
\hline 4 & Male & 16 & 180 & 73.2 & 22.6 & 4137 & n.d & $<107.1$ & $<230.4$ \\
\hline 5 & Female & 15 & 167 & 63.2 & 22.7 & 2714 & n.d & $<124.4$ & $<257.2$ \\
\hline 6 & Female & 17 & 174 & 75.5 & 24.9 & 3068 & $<47.2$ & $<123.6$ & $<264.9$ \\
\hline 7 & Female & 17 & 170 & 66.0 & 22.8 & 2931 & $<42.9$ & $<119.3$ & $<261.7$ \\
\hline 8 & Female & 16 & 177 & 74.8 & 23.9 & 3330 & $<44.2$ & $<121.7$ & $<257.9$ \\
\hline 9 & Female & 17 & 166 & 68.0 & 24.7 & 3038 & $<46.8$ & $<124.2$ & $<265.9$ \\
\hline Mean & & 17.3 & 177 & 73.8 & 23.6 & 3894 & 43.0 & 115.1 & 245.5 \\
\hline SD & & 2.3 & 9 & 7.7 & 1.0 & 1110 & 4.0 & 9.0 & 19.3 \\
\hline
\end{tabular}

peak $\mathrm{HR}\left(\mathrm{HR}_{\text {peak }}\right)$ to $\mathrm{HR}$ at $2.5 \mathrm{mmol} \mathrm{L}^{-1}$ blood lactate; $\mathrm{Z} 2$ with HR and velocity corresponding to $2.5-4.0 \mathrm{mmol} \mathrm{L}^{-1}$ blood lactate and Z3 as an intensity with HR and velocity corresponding above $4.0 \mathrm{mmol} \mathrm{L}^{-1}$ blood lactate. The 2.5 and $4 \mathrm{mmol} \mathrm{L}^{-1}$ reference points refer to training zone prescriptions of the German Canoe Federation [14].

The TID based on race pace was also based on a threezone model and based on previous categorization $[7,8]$ with $\mathrm{Z} 1$ as velocity corresponding from $60 \% \mathrm{HR}_{\text {peak }}$ to $85 \%$ of race pace, $Z 2$ as velocity corresponding from 86 to $95 \%$ of race pace and $\mathrm{Z} 3$ as velocity corresponding above $95 \%$ of race pace. These zone demarcations correspond to training recommendations by the Germany Canoe Federation for the three zones [14].

\section{Polarization-Index}

To quantify the individual level of polarization, we calculated a Polarization-Index (Pol-Index; a.u.) based on the time trained in each intensity zone. The Pol-Index was calculated as described in detail previously $[15,16]$ and calculated as follows:

$$
\text { Polarization-Index (a.u.) }=\log _{10}(\mathrm{Z} 1 / \mathrm{Z} 2 * \mathrm{Z3} * 100) \text {. }
$$

A Pol-Index of $>2.0$ a.u. is proposed to reflect a polarized TID.

\section{Incremental Testing}

All on-water testing took place on the regatta course in Duisburg-Wedau (Germany), the venue for several international canoe sprint championships, as well as the World Cup series of the International Canoe Federation (ICF). The participants were asked to refrain from all physical exercise for $12 \mathrm{~h}$ and exhausting exercise for $48 \mathrm{~h}$ prior to the experimental sessions, as well as to maintain their regular diet. In addition, all were instructed to avoid food intake $2 \mathrm{~h}$ prior to testing and were requested to arrive in a well hydrated state. Their diet and state of hydration were assessed with pre-test questionnaires.

The incremental test protocol involved $4 \times 1500-\mathrm{m}$ trials on-water at different intensities (i.e. 70\%, 80\% and $90 \%$ of $\mathrm{HR}_{\text {peak }}$, as well as an all-out effort) as described in detail previously [17]. Each incremental step was performed with a turn at $750 \mathrm{~m}$ to weaken the influence of wind and waves on performance. The time for each change in direction was extracted using the GPS-data. Previous investigations involving on-water incremental step testing in sprint kayakers showed acceptable to excellent reliability and validity of measurements of $\mathrm{HR}$, oxygen uptake, blood lactate and stroke rate $[18,19]$ and strong correlations with performance [17].

The HR utilized for the first test was based on $\mathrm{HR}_{\text {peak }}$ obtained 6 to 8 weeks before with the same incremental on-water test protocol by the Western German Canoe Federation and each following test utilized the maximal heart rate of the previous testing. The 30-45 s interval between successive steps was the time required to sample capillary blood from the earlobe. All participants received continuous visual feedback from their HR monitor (Polar Wear Link System and V800 HR Monitor, Polar Electro OY, Kempele, Finland) mounted directly in front of them and averaged every second. The highest value during each incremental test was considered as $\mathrm{HR}_{\text {peak }}$. During each stage the stroke rate was self-selected.

Capillary blood was sampled from the right earlobe for analysis of lactate (Lactate Pro 2, Arkray KDK, Kyoto, Japan), at baseline and after each step. The HR and velocity associated with corresponding blood lactate 
concentrations were determined by linear interpolation between the two closest points, as in previous studies [20]. At the same time-points, rating of perceived exertion (RPE) was assessed employing the 6-20-point Borg scale [21].

\section{Heart Rate and Velocity Monitoring}

The HR, velocity, distance and duration of every training session were collected from each sprint kayaker with a GPS-enabled watch (M430, Polar Electro Oy, Kempele, Finland) and the data were stored online (Polar Flow, Polar Electro Oy). To maximize measurement accuracy, HR was measured using a HR chest strap connected with the M430. All initiated the recording of each session with the beginning of the warm-up and stopped recording immediately with completion of cool-down. Afterwards, all datasets were visually checked for artefacts (e.g. flatline). Incomplete data files due to technical issues (e.g. low battery, HR or GPS-inaccuracy, etc.) and excluded from further analysis. Additionally, an experienced coach compared the number of recorded sessions with the online training diary of each athlete. From the data provided by the online version of the HR monitor (Polar Flow software), the total time spent in each HR and velocity zone for each training session was calculated.

\section{Statistics}

SPSS Statistics (Version 26; IBM Corp., Armonk, NY) was utilized for all statistical analysis. As performed earlier [6] a two-way analysis of variance with Bonferroni post hoc test was implemented to analyse for differences between TIDs derived from HR and velocity based on blood lactate-based zone demarcation as well as velocity based on race pace-based zone demarcation. Statistical significance was defined as $p \leq 0.05$. Effect sizes were calculated as Cohen's $d$ values (Cohen, 1988), where 0.000.41 represents a small, $0.41-0.70$ a moderate and $\geq 0.70$ a large effect. In addition, 95\% confidence intervals (95\% CI) for Cohen's $d$ were calculated.

\section{Results}

During the 8-week observation period, 557 single sessions with approximately $4500 \mathrm{~km}$ or $500 \mathrm{~h}$ of training were incorporated for analysis. The average training time per week within this period was $13.3 \pm 1.4 \mathrm{~h}$, with $7.9 \pm 1.2 \mathrm{~h}$ of kayak-specific on-water training, $5.2 \pm 0.7 \mathrm{~h}$ strength training, $1.8 \pm 0.7 \mathrm{~h}$ other endurance training (e.g. running, swimming, cycling) and $1.1 \pm 0.5 \mathrm{~h}$ of other activities (e.g. stretching, yoga).

The mean TID for the three different methods in each training zone during the observation period is summarized in Fig. 2. Figure 3 displays the TID for each participant. Additionally, Fig. 4 shows the




Pol-Index for each participant and each TID determination method.

For TID $_{\text {Race }}$ analysis, the mean velocity for the upper limit of $\mathrm{Z} 1$ and $\mathrm{Z} 2$ was $11.80 \pm 0.94 \mathrm{~km} \cdot \mathrm{h}^{-1}$ and $13.27 \pm 1.06 \mathrm{~km} \cdot \mathrm{h}^{-1}$, respectively. For the $\mathrm{TID}_{\text {Bla-HR }}$ and $\mathrm{TID}_{\text {Bla- } \mathrm{V}}$ the upper limits were $12.37 \pm 0.80 \mathrm{~km} \mathrm{~h}^{-1}$ (Z1) and $13.02 \pm 0.82 \mathrm{~km} \cdot \mathrm{h}^{-1}$ (Z2). Mean HR for the upper limit of $\mathrm{Z} 1$ and $\mathrm{Z} 2$ for $\mathrm{TID}_{\mathrm{Bla}-\mathrm{HR}}$ was $173 \pm 9$ and $183 \pm 10$ beats per minute.

When employing $\mathrm{TID}_{\mathrm{Bla}-\mathrm{HR}}$ the mean plural of $\mathrm{Z1}$, $\mathrm{Z} 2$ and $\mathrm{Z} 3$ were $91 \pm 4 \%, 6 \pm 2 \%$ and $3 \pm 2 \%$ (Pol-Index: $1.6 \pm 0.4)$. For $\mathrm{TID}_{\mathrm{Bla}-\mathrm{V}}$ the fractions were $68 \pm 14 \%$, $14 \pm 8 \%$ and $18 \pm 8 \%$ (Pol-Index: $1.9 \pm 0.3$ ), with each fraction differing to $\mathrm{TID}_{\mathrm{Bla}-\mathrm{HR}}(\mathrm{Z} 1: p<0.01, d=-2.23$, 95\% CI -3.28 to $-0.97 ; \mathrm{Z2}: p=0.01, d=1.37,95 \%$ CI0.29 to 2.32; Z3: $p<0.01, d=2.57,95 \%$ CI 1.23 to 3.67). The $\mathrm{Z} 1, \mathrm{Z} 2$ and $\mathrm{Z} 3$ plural of $\mathrm{TID}_{\text {Race }}$ were $73 \pm 5 \%, 20 \pm 5 \%$ and $7 \pm 2 \%$ (Pol-Index: $1.4 \pm 0.2$ ), respectively. $\mathrm{Z} 1$ and $\mathrm{Z} 2$, but not $\mathrm{Z3}$ of $\mathrm{TID}_{\text {Race }}$ zone fractions differed when compared to $\mathrm{TID}_{\mathrm{Bla}-\mathrm{HR}}(\mathrm{Z} 1$ : $p<0.01, d=4.20,95 \% \mathrm{CI} 2.40$ to 5.60 ; Z2: $p<0.01$, $d=-3.68,95 \% \mathrm{CI}-4.97$ to -2.03$)$ and compared to $\mathrm{TID}_{\text {Bla-V }}$ only Z3 was different $(p<0.01, d=1.89,95 \%$ CI 0.70 to 2.89 ).

On an individual level the ranges in each zone and with each TID determination method were as follows: For $\mathrm{TID}_{\text {Bla-HR }} \mathrm{Z} 1$ to $\mathrm{Z} 3$ ranged from 85 to $98 \%, 2$ to $11 \%$ and 0.1 to $6 \%$ (Pol-Index: 0.3-2.1), for TID Bla-V $_{4} 41$ to $82 \%, 6$ to $30 \%, 8$ to $30 \%$ (Pol-Index: $1.6-2.4$ ) and for TID $_{\text {Race }} 64$ to $81 \%, 14$ to $29 \%$ and $4-10 \%$ (Pol-Index: 1.2-1.7), respectively. With $\mathrm{TID}_{\text {Bla-HR }}$ all but one of the athletes revealed a pyramidal TID (Pol-Index $<2.0$ ) with extremely high fractions in Z1 (> 85\%), while only one athlete (P-ID 2) highlighted a polarized TID with a Pol-Index of 2.1 and only $2 \%$ difference between Z2 (4\%) and Z3 (6\%). With TID Bla-V three athletes (P-ID $2,5,7)$ showed a polarized intensity distribution (PolIndex $\geq 2.1$ ) and two athletes revealed a polarized like TID pattern, i.e. higher proportions of Z3 than Z2, with one of the two (P-ID 6; Pol-Index $=1.9$ ) revealing almost identical fractions for Z2 (11\%) and Z3 (13\%) and the other (P-ID 4; Pol-Index $=1.6$ ) relatively low proportion in Z1 (55\%). Additionally, one athlete (P-ID 9; Pol-Index: 1.6) with TID $_{\text {Bla-V }}$ had an almost uniform distribution of the three zones with 40,30 and $30 \%$ in

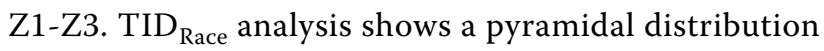
for each athlete.

\section{Discussion}

The current study aimed to retrospectively compare different TID quantification methods based on physiologically determined intensity zones using HR and velocity monitoring as well as race pace-based zone determination using velocity monitoring during an 8-week competition period in highly trained kayak sprint athletes. This retrospective analysis is to best of our knowledge the first observation in the literature comparing these different quantification methods for TID in kayak sprinting.

The major findings were:

(i) $\mathrm{TID}_{\mathrm{Bla}-\mathrm{HR}}$ significantly differed in each intensity zone from $\mathrm{TID}_{\mathrm{Bla}-\mathrm{V}}$ and in $\mathrm{Z} 1$ and $\mathrm{Z} 2$ from $\mathrm{TID}_{\text {Race }}$

(ii) $\mathrm{TID}_{\mathrm{Bla}-\mathrm{V}}$ differed in $\mathrm{Z} 3$ fractions when compared TID Race

(iii) Depending on the quantification method and on group level $\mathrm{TID}_{\mathrm{Bla}-\mathrm{HR}}$ and $\mathrm{TID}_{\text {Race }}$ yielded rather pyramidal TID whereas $\mathrm{TID}_{\mathrm{Bla}-\mathrm{V}}$ resulted in more polarized TID.

(iv) On an individual level $\operatorname{TID}_{\text {Race }}$ was pyramidal for each athlete, $\mathrm{TID}_{\mathrm{Bla}-\mathrm{HR}}$ was pyramidal for eight athletes and polarized for one athlete and TID $_{\text {Bla-V }}$ revealed to be polarized for three athletes, polarized like for two athletes, uniform for one and pyramidal for three athletes.

\section{Intensity Distribution Based on $\mathrm{TID}_{\mathrm{Bla}-\mathrm{HR}}$ and $\mathrm{TID}_{\text {Bla-V }}$}

The fraction of each zone with $\mathrm{TID}_{\mathrm{Bla}-\mathrm{HR}}$ and $\mathrm{TID}_{\mathrm{Bla}-\mathrm{V}}$ differed substantially. $\mathrm{TID}_{\mathrm{Bla}-\mathrm{HR}}$ revealing a pyramidal TID for Z1, Z2 and Z3 $(91 \pm 4 \%, 6 \pm 2 \%, 3 \pm 2 \%$; Pol-Index: $1.6 \pm 0.4)$, whereas $\mathrm{TID}_{\text {Bla-V }}$ showed a more polarized TID (68 $\pm 14 \%, 14 \pm 8 \%$ and $18 \pm 8 \%$; Pol-Index: $1.9 \pm 0.3$ ). Research concerning both TID quantifications methods is scarce, and so far, the only existing studies were implemented in running [6] and cycling [22], e.g. endurance sports that mainly implement the lower body for propulsion. In contrast, sprint kayaking imposes high demands on the endurance capacity of the athletes' upper body, as the athlete propels the boat-body-system against water resistance involving relatively small upper-body muscles [10]. Differences in muscle mass, muscle fibre spectrum, oxygen extraction [11] as well as glucose and lipid oxidative capacity [11-13] between upper and lower body require different qualitative and quantitative training stimuli for distinct adaptation. Recent studies in elite cross-country skiers investigated upper and lower-body muscles and found

(See figure on next page.)

Fig. 3 Training intensity distribution (percentage of total training time spent in each training zone) for each participant where zones were determined by heart rate $\left(\mathrm{TID}_{\mathrm{Bla}-\mathrm{HR}}\right)$ or velocity $\left(\mathrm{TID}_{\mathrm{Bla}-\mathrm{V}}\right)$ according to physiological testing and by velocity according to percentages from race pace $\left(T I D_{\text {Race }}\right)$ 

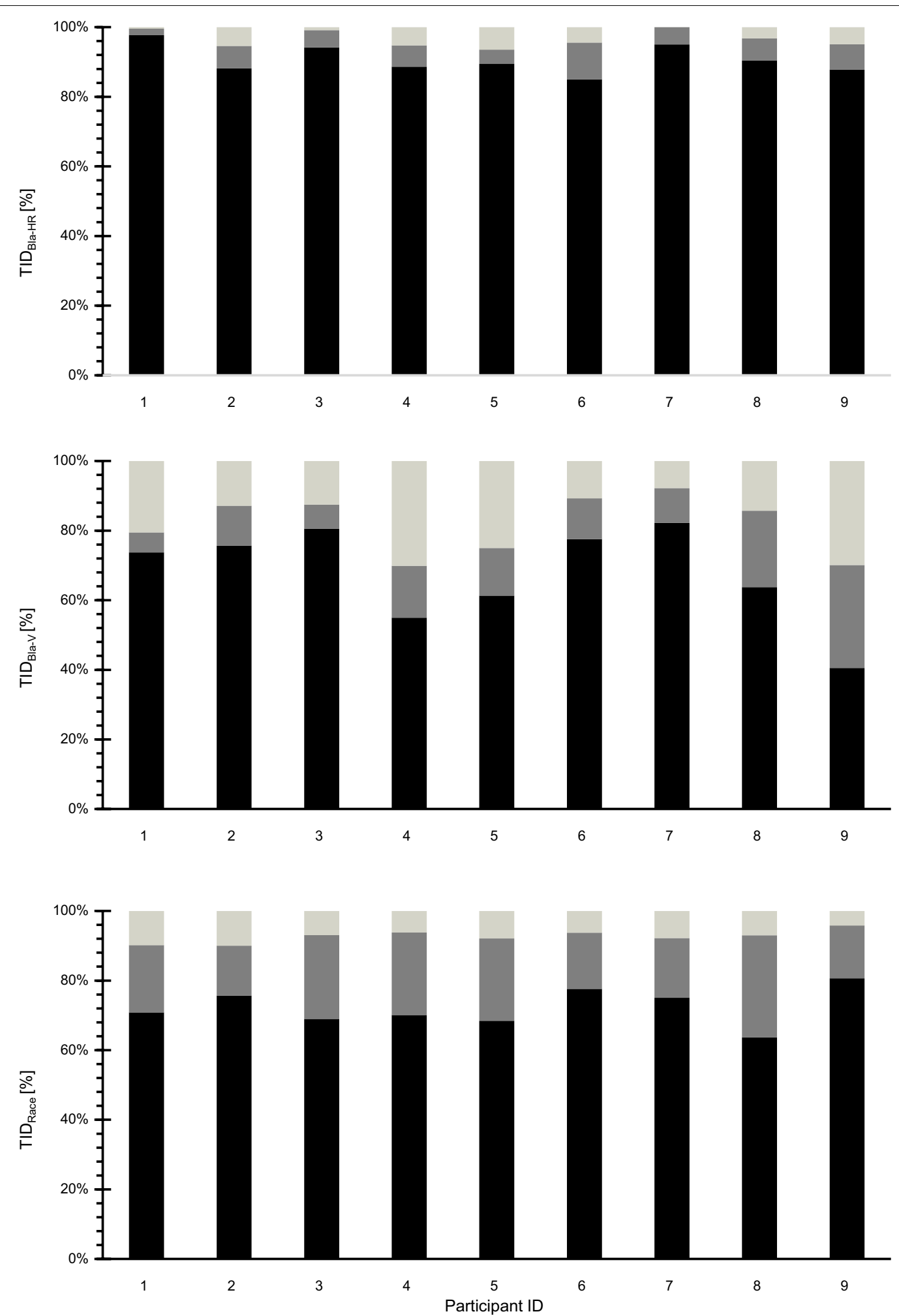

$\mathrm{TID}[\mathrm{Bla}-\mathrm{HR}]=$ Training intensity distribution determined by heart rate according to blood lactate-based zone demarcation from incremental test

$\mathrm{TID}[\mathrm{Bla}-\mathrm{V}]=$ Training intensity distribution determined by velocity according to blood lactate-based zone demarcation from incremental test

TID $[$ Race $]=$ Training intensity distribution determined by velocity according to percentages from race-pace 


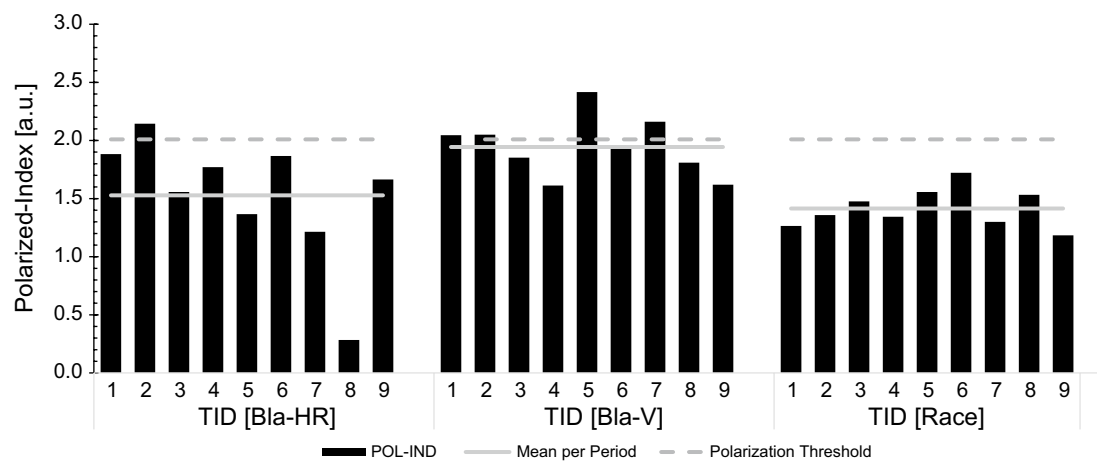

TID $[\mathrm{Bla}-\mathrm{HR}]=$ Training intensity distribution determined by heart rate according to blood lactate-based zone demarcation from incremental test TID $[\mathrm{Bla}-\mathrm{V}]=$ Training intensity distribution determined by velocity according to blood lactate-based zone demarcation from incremental test TID $[$ Race $]=$ Training intensity distribution determined by velocity according to percentages from race-pace POL-IND = Polarisation Index

Fig. 4 Individual Polarization-Index (POL-IND) for each analysed macrocycle and athlete. Mean=Group mean of Polarization-Index for the particular parameter. Polarization Threshold = Above a value of 2.0 in the Polarization-Index the TID is polarized (Treff et al., 2019)

upper-body muscles to be less capable to oxidize fat and to rely more on carbohydrate oxidation than lower-body muscles $[11,12]$. Thus, it may seem plausible to assume that kayakers may not be able to perform as much volume in the higher intensity zones (Z2, Z3), as arm-glucose storages depleted earlier and therefore kayakers (have to) implement more time in Z1 compared to whole-body and/or lower-body sports.

Despite the differences in muscles involved in propulsion, data from endurance running [6] and endurance cycling [22] are partly in agreement with the current findings. Bellinger et al. analysed the TID based on HR and running speed corresponding to below first ventilatory threshold, between first and second ventilatory threshold and above second ventilatory threshold of fourteen highly trained middle-distance runners during an 8-wk training period [6]. In the latter investigation, TID differed especially in time spent in Z2 (HR: $17.0 \pm 6.3 \%$ vs. speed: $5.3 \pm 4.9 \%$ ) and Z3 (HR: $3.4 \pm 2.0 \%$ vs. speed: $14.7 \pm 7.3 \%)$, resulting in a polarized TID for the velocitybased and a pyramidal TID for HR-based zone quantification. In line with the results of Bellinger and colleagues [6], another study [22] found higher fractions of Z3 for power output-based TID (11.5 $\pm 2.5 \%)$ quantification compared to HR-based TID quantification (4.4 $\pm 2.0 \%)$, when comparing a three-zone TID model based on aerobic and anaerobic threshold performance in road cyclists during a 10 -week training period.

The differences in $\mathrm{Z} 2$ and $\mathrm{Z} 3$ between HR- and velocity/power-derived TID quantification shown in the previous studies $[6,22]$ and our findings between TID $_{\text {Bla-HR }}$ and TID $_{\mathrm{Bla}-\mathrm{V}}$ are most likely attributable to the delayed HR-kinetics at the beginning of an (intense) exercise bout. Especially, with short high-intensity bouts $(<30 \mathrm{~s})$ the HR response is inertial and does not reflect the entire 30-s effort [5, 23]. Thus, lower fractions of $\mathrm{Z} 3$ and higher fractions of $\mathrm{Z} 2$ with $\mathrm{TID}_{\mathrm{Bla}-\mathrm{HR}}$ are possible. As a consequence, TID analysis based on HR is likely impractical to reflect the neuromuscular demand of high-intensity short-duration efforts, while external measures such as velocity seem to reflect these demands more accurately $[22,23]$. In fact, in training regimes of sprint kayaking short intervals with maximum intensity are frequently implemented as the (i) start phase performance has an important impact on race performance [10] and (ii) work performed during $30 \mathrm{~s}$ all-out kayaking is correlated with performance over all three Olympic distances [24] as reflected in the higher fractions of $\mathrm{Z} 3$ for velocity-based measures in the current study. The underestimation of Z3 by HR-monitoring is further confirmed by two recent studies [25, 26] investigating kayak sprinters and comparing post-session intensity analysis based on $\mathrm{HR}$ and power output. In this case, HR-based training monitoring also underestimated high-intensity training loads and overestimated training time in Z2. Accordingly, in phases with focus on lower intensities and longer distances covered in each exercise bout (e.g. general preparatory period) TID would differ less between HR and external measures compared to phases with more short highintensity training sessions (e.g. competition and taper periods) [22].

In the present analysis quantification of $\mathrm{Z} 1$ markedly differed to the findings of previous studies [6, 22]. The current study shows significantly higher proportions of $\mathrm{Z} 1 \mathrm{TID}_{\mathrm{Bla}-\mathrm{HR}}$ compared to $\mathrm{TID}_{\mathrm{Bla}-\mathrm{V}}$, while Bellinger et al. [6] and Sanders et al. [22] found no difference for $\mathrm{Z} 1$ fractions between $\mathrm{HR}$ and velocity monitoring. The following explanation may account for the inconsistent 
data: kayakers often perform their long-distance endurance sessions in groups and usually one kayaker leads, and the training partners follow situated to the left and right side behind the leader and/or directly behind him/ her [27]. This arrangement is called "wash-riding" as the kayakers behind the leader are assisted in their forward paddling by the force of the "wash" (i.e. undertow) generated by the boat of the leading kayaker. Previous analysis found that wash-riding saves up to $31.9 \%$ of energy, which is also associated with markedly decreased blood lactate and heart rate values during a constant endurance session [27]. As it is common for kayakers to change the group leader each kilometre or each $5 \mathrm{~min}$, the position alterations allow the group to maintain a relatively high velocity over an entire session reflecting a Z2-velocity while keeping the HR low (i.e. Z1). Therefore, from a practical point of view, it is likely that $\mathrm{Z} 1$ contribution is underrated and Z2 contribution overrated when employing TID $_{\text {Bla-V}}$. Future studies on TID in kayaking should consider carefully, if the athletes should be allowed to perform wash-riding.

Consequently, when analysing training intensity in kayak sprinters, both quantification methods TID $_{\text {Bla-HR }}$ and $\mathrm{TID}_{\mathrm{Bla}-\mathrm{V}}$ may have crucial downsides for TID analysis and further decision making. Based on the present data, a combination of both methods may yield value for TID quantification in kayak sprinting with $\mathrm{TID}_{\mathrm{Bla}-\mathrm{HR}}$ implemented to determine $\mathrm{Z} 1$ and $\mathrm{Z} 2$ and $\mathrm{TID}_{\mathrm{Bla}-\mathrm{V}}$ for $\mathrm{Z} 3$. Further research is warranted in this regard.

In summary, previous retrospective $\mathrm{TID}_{\mathrm{Bla}-\mathrm{HR}}$ analysis seems to yield more pyramidal TID [3, 6, 28-33] whereas when integrating external measurement (e.g. velocity or power output) [6] or the session goal method [3,34] with complemented HR measurement the TID reveals to be more polarized. This pattern of TID-dependence on the implemented quantification method applies over a range of different sports like running $[6,28]$, cycling $[29,31$, 33], cross-country skiing [3], rowing [30].

\section{Intensity Distribution Based on TID $_{\text {Race }}$}

The analysis of TID $_{\text {Race }}$ showed a pyramidal TID pattern on the group level. Most interestingly, this pattern was found consistently for each athlete (Fig. 3C). This high congruency among the group in TID was not evident with the other two quantification methods, i.e. $\mathrm{TID}_{\text {Bla-HR }}$ and TID $_{\text {Bla-V }}$ (Fig. 3A, B). Similarly, Kenneally et al. [7] concluded that TID is subject to less interindividual variation among middle- and long-distance runners when employing race pace compared to the physiological benchmark-based TID quantification, probably because training during the competitive period targets mainly to develop race pace. In a review article, the same research group [8] analysed TID of different middle- and long-distance runners employing either HRor velocity-based TID and calculated training intensity zones relative to the targeted race pace and found race pace to be a more important factor in the design of training programmes than physiological zone demarcation and founded this in the high similarity between athletes TID when based on race pace.

However, it remains questionable if the consistency in $\mathrm{TID}_{\text {Race }}$ zone proportion among athletes favours argument for race pace-based zone quantification, as, e.g. so far, no study analysed coherences between race pace-based zone quantification and performance-related measures. Additionally, the $\mathrm{TID}_{\text {Race }}$ in our analysis resulted in a markedly wider speed range for $\mathrm{Z} 2(1.47 \mathrm{~km} / \mathrm{h} \pm 0.12 \mathrm{~km} / \mathrm{h})$ when compared with the physiologically based $\mathrm{TID}_{\mathrm{Bla}-\mathrm{HR}}$ and $\mathrm{TID}_{\mathrm{Bla}-\mathrm{V}}$ $(0.66 \mathrm{~km} / \mathrm{h} \pm 0.27 \mathrm{~km} / \mathrm{h})$, which is in accordance with previous research $[7,35]$ and most probably explains the higher homogeneity of $\mathrm{TID}_{\text {Race }}$. The markedly wider speed range for $Z 2$ in $\mathrm{TID}_{\text {Race }}$ explains the shift from higher fractions in $\mathrm{Z} 3$ and lower fractions in $\mathrm{Z} 2$ in the physiological approach to comparably higher fractions in $\mathrm{Z} 2$ and lower fractions in $\mathrm{Z} 3$ compared to the race pace approach, which was similarly found in middle- and longdistance runners [7]. The wide range of $\mathrm{Z} 2$ in the race pace approach certainly includes intensities that exceed the "threshold" zone and thus, possibly provides a wider range of training stimuli [7]. If coaches are not aware of the higher intensities included in $\mathrm{Z} 2$ when using the current approach of race pace-based training intensity prescription, the use of the method potentially may lead to an unintended overload. Although the TID $_{\text {Race }}$ assists coaches and athletes with feedback about the training intensity relative to competition performance (and therefore representing a marker with high specificity especially in the final weeks prior to competitions), the TID analyses based on physiological zone demarcation is important to receive knowledge about the development of the different energy systems during the training process.

Furthermore, especially for the sport of sprint kayaking, TID $_{\text {Race }}$ reveals several difficulties: (i) as an outdoor sport, kayaking race performance is strongly affected by weather conditions (e.g. water temperature, wind, waves, etc.) and type of waters (e.g. artificial regatta course, lake, river, etc.) where training and competition are performed, leading to substantial variation in performance times. This variation subjects a session-to-session as well as competition-to-competition comparison of speed sensitive to errors. Future research should provide recommendations for kayak-specific training intensity prescription/analysis which consider these variations. (ii) In kayaking it is quite common to train for and compete over, at least, two of the three Olympic distances $(200 \mathrm{~m}$, 
$500 \mathrm{~m}, 1000 \mathrm{~m}$ ). This variation can lead to differing intensity prescriptions using race pace, which most probably is no issue within $\mathrm{Z3}$ sessions, where the session aim is mostly specific to the race distance. However, during $\mathrm{Z} 1$ and $\mathrm{Z} 2$ sessions, the session aim must not be clearly assigned to a specific race distance. As, in the current study, race pace-based TID was solely based on $1000 \mathrm{~m}$ race pace, $Z 3$ fractions would have been even lower in favour of $\mathrm{Z} 2$ if each sessions intensity would have been calculated on the race distance targeted in the individual session. For example, an athlete with a race pace of $15.00 \mathrm{~km} / \mathrm{h}(=4: 00 \mathrm{~min} / \mathrm{km})$ over the $1000-\mathrm{m}$ distance and of $16.36 \mathrm{~km} / \mathrm{h}(=3: 40 \mathrm{~min} / \mathrm{km})$ over the $500-\mathrm{m}$ distance, who trains 2-min-intervals at a pace of $14.50 \mathrm{~km} / \mathrm{h}$ $(=4: 08 \mathrm{~min} / \mathrm{km})$, would have a Z3 session when analysing based on $1000-\mathrm{m}$ race pace $(14.50 \mathrm{~km} / \mathrm{h}=97 \%$ of $1000-\mathrm{m}$ race pace), but only a $\mathrm{Z} 2$ session when $500-\mathrm{m}$ race pace $(14.50 \mathrm{~km} / \mathrm{h}=89 \%$ of $500 \mathrm{~m}$ race pace) is the benchmark for analysis. A possible compromise could be to use the race pace of the main competition which an athlete is primarily focusing on. If an athlete competes in two different disciplines (e.g. $200 \mathrm{~m}$ and $500 \mathrm{~m}$ ), one might consider using the race pace of an intermediate distance (e.g. $350 \mathrm{~m}$ ) to provide a TID quantification. A further method could be the training intensity prescription and/or analysis based on the individual session aim (e.g. the individual race pace of the targeted race distance in each session). This approach has been discussed previously [23] and focusses on the training of the athletes' entire locomotor profile (e.g. start phase performance and anaerobic speed) instead of solely focusing on developing a single physiological performance parameter (e.g. speed at anaerobic threshold). As competition performance is determined by the interaction of different performance-related parameters [24], this method, oriented on the race distance targeted in the individual session, may provide a more goal-orientated approach compared to training prescription solely based on physiological parameters. However, this method is suggested to be mostly valuable for experienced athletes and coaches who know the best performance times on several distances and have low intersession performance variation [23]. Ultimately, in contrast to $\mathrm{TID}_{\mathrm{Bla}-\mathrm{HR}}$ and $\mathrm{TID}_{\mathrm{Bla}-\mathrm{V}}$, the aforementioned examples for adjusting the quantification of TID $_{\text {Race }}$ demonstrate the flexibility of this method for adapting specific aims and circumstances of each athlete in his/her preparation.

Since TID $_{\text {Bla-HR }}$, TID $\mathrm{Bla}_{\mathrm{Bl}-\mathrm{V}}$ and TID $_{\text {Race }}$ demonstrate upand downsides it appears important for researchers and coaches to raise the question about "what is the main aim of the current training phase?". The race pace-based approach might be of value during competition periods, but during periods with less emphasis on specific race pace development the threshold-based approach might be more valuable. Additionally, a shift from a TID $_{H R}$ and TID $_{\text {Bla }}$ during preparatory periods to a race pace-based approach during the competition period would be in accordance with one of the most accepted principles of training, which is the principle of specific adaptations to the imposed demands [36] (SAID-principle). The SAIDprinciple considers the versatility of influences, beside the traditional metabolic considerations, that impact race performance. Especially, as until today, research failed to find the one optimal TID model and/or physiological parameter to predict performance [3, 8], race pace-based intensity prescription may provide a better stimulus to concurrently develop key endurance variables in the final preparation for competition performance [8].

\section{Limitations}

Certain limitations associated with the current investigation warrant consideration. The relatively small number of subjects may have affected the power of the statistical analysis. However, it is difficult to conduct investigations of this nature on large numbers of highly trained athletes who must prioritize their own individual training and competition. We believe that the current investigation provides useful information for coaches and researchers in this specific field. Moreover, it is important to note that on-water TID analysis does not provide a comprehensive evaluation of the stress an athlete is exposed to. Non-specific training (e.g. resistance training, dry-land endurance training, etc.) and various additional factors (e.g. sleep, daily physical activity, nutrition, etc.) have an impact on overall training stress and need to be considered when holistically evaluating an athletes training stress [37, 38]. Furthermore, as discussed earlier, when analysing data from an outdoor sport like kayaking, one must be aware of the unstable nature of the sport that is constantly influenced by water and weather conditions. Thus, a future challenge for research is to provide models to minimize the impact of environmental fluctuations on TID analysis.

\section{Conclusion}

The current study aimed to retrospectively compare TID quantification based on physiologically determined intensity zones as well as race pace-based zone determination using velocity monitoring during the competition period in highly trained kayak sprint athletes. Based on the present data and observation period we conclude that the method of quantification affects the calculation of the TID in highly trained sprint kayakers. The most noticeable difference between the TID determination method is the comparably low interindividual variation in TID for $\mathrm{TID}_{\text {Race }}$. Depending on the aim of the analysis and 
training goal $\mathrm{TID}_{\text {Race, }}, \mathrm{TID}_{\mathrm{Bla}-\mathrm{HR}}$ and $\mathrm{TID}_{\mathrm{Bla}-\mathrm{V}}$ have advantages as well as drawbacks and may be implemented in conjunction to maximize adaptation.

\begin{abstract}
Abbreviations
a.u.: Arbitrary unit; $\mathrm{B}_{\mathrm{la}}$ : Blood lactate; Cl: Confidence interval; GPS: Global position system; P-ID: Participant identification; Pol-Index: Polarization-Index; RPE: Rating of perceived exertion; TID: Training intensity distribution; $\mathrm{TID}_{\mathrm{Bla}-}$ H: Training intensity distribution derived from heart rate based on blood lactate concentrations of incremental step testing; $\mathrm{TI}_{\text {Bla-v }}$ : Training intensity distribution derived from velocity based on blood lactate concentrations of incremental step testing; $T I D_{\text {Race }}$ :Training intensity distribution derived from velocity based on 1000-m race pace; $H R$ : Heart rate; $\mathrm{HR}_{\text {peak }}$ : Peak heart rate; $\mathrm{VO}_{2}$ : Oxygen uptake; $\mathrm{VO}_{2 \text { max }}$ : Maximum oxygen uptake; Z: Zone.
\end{abstract}

\section{Acknowledgements}

The authors would like to thank all the athletes and coaches for their cooperation and for the time they dedicated to this study.

\section{Authors' contributions}

All authors contributed to the study conception and design. Material preparation, data collection and analysis were performed by MM, CZ and BS. Data were exported and processed by RL. The first draft of the manuscript was written by MM. MM, CZ and BS commented on previous versions of the manuscript. All authors read and approved the final manuscript.

\section{Funding}

The project was supported financially by the German Federal Institute of Sports Sciences.

\section{Availability of Data and Materials}

As the athletes in this study are still competing on national and international level, supporting data are not available.

\section{Declarations}

\section{Ethics Approval and Consent to Participate}

This study was approved by the Ethics Committee of the Julius-Maximilians University of Würzburg and conforms to the Declaration of Helsinki. Prior to participation, written informed consent was obtained.

\section{Consent for Publication}

Not applicable.

\section{Competing Interests}

Billy Sperlich, Christoph Zinner, Manuel Matzka and Robert Leppich declare that they have no competing interests.

\section{Author details \\ ${ }^{1}$ Integrative and Experimental Exercise Science and Training, University of Würzburg, Würzburg, Germany. ${ }^{2}$ Software Engineering Group, Department of Computer Science, University of Würzburg, Würzburg, Germany. ${ }^{3}$ Depart- ment of Sport, University of Applied Sciences for Police and Administration of Hesse, Wiesbaden, Germany.}

Received: 5 August 2021 Accepted: 14 November 2021

Published online: 06 January 2022

\section{References}

1. Esteve-Lanao J, Foster C, Seiler S, Lucia A. Impact of training intensity distribution on performance in endurance athletes. J Strength Cond Res. 2007:21:943-9.

2. Mujika I, Chatard JC, Busso T, Geyssant A, Barale F, Lacoste L. Effects of training on performance in competitive swimming. Can J Appl Physiol Rev Can Physiol Appl. 1995;20:395-406.
3. Seiler S, Kjerland G. Quantifying training intensity distribution in elite endurance athletes: is there evidence for an „optimal“ distribution? Scand J Med Sci Sports. 2006;16:49-56.

4. Stöggl T, Sperlich B. The training intensity distribution among well-trained and elite endurance athletes. Front Physiol. 2015;6:295.

5. Seiler $\mathrm{S}$. What is best practice for training intensity and duration distribution in endurance athletes? Int J Sports Physiol Perform. 2010;5:276-91.

6. Bellinger $P$, Arnold B, Minahan C. Quantifying the training-intensity distribution in middle-distance runners: the influence of different methods of training-intensity quantification. Int J Sports Physiol Perform. 2020;15:319-23.

7. Kenneally M, Casado A, Gomez-Ezeiza J, Santos-Concejero J. Training intensity distribution analysis by race pace vs. physiological approach in world-class middle- and long-distance runners. Eur J Sport Sci. 2021;21:819-26.

8. Kenneally M, Casado A, Santos-Concejero J. The effect of periodization and training intensity distribution on middle- and long-distance running performance: a systematic review. Int I Sports Physiol Perform. 2018;13:1114-21

9. Stögg|T, Sperlich B. Polarized training has greater impact on key endurance variables than threshold, high intensity, or high volume training. Exerc Physiol. 2014;5:33.

10. Ualí I, Herrero AJ, Garatachea N, Marín PJ, Alvear-Ordenes I, García-López D. Maximal strength on different resistance training rowing exercises predicts start phase performance in elite kayakers. J Strength Cond Res. 2012;26:941-6.

11. Calbet JAL, Holmberg H-C, Rosdahl H, van Hall G, Jensen-Urstad M, Saltin B. Why do arms extract less oxygen than legs during exercise? Am J Physiol-Regul Integr Comp Physiol. 2005;289:R1448-558.

12. Ørtenblad N, Nielsen J, Boushel R, Söderlund K, Saltin B, Holmberg H-C. The muscle fiber profiles, mitochondrial content, and enzyme activities of the exceptionally well-trained arm and leg muscles of elite cross-country skiers. Front Physiol. 2018. https://doi.org/10.3389/fphys.2018.01031/full.

13. Zinner C, Morales-Alamo D, Ørtenblad N, Larsen FJ, Schiffer TA, Willis SJ, Gelabert-Rebato M, Perez-Valera M, Boushel R, Calbet JA, Holmberg HC. The physiological mechanisms of performance enhancement with sprint interval training differ between the upper and lower extremities in humans. Front Physiol. 2016;7:426.

14. Kahl J. DKV-Rahmentrainingskonzeption-Kanurennsport und Kanuslalom. Duisburg Dtsch Kanu-Verband-Wirtsch-Verl GmbH. 2005.

15. Treff G, Winkert K, Sareban M, Steinacker JM, Becker M, Sperlich B. Elevenweek preparation involving polarized intensity distribution is not superior to pyramidal distribution in national elite rowers. Front Physiol. 2017. https://doi.org/10.3389/fphys.2017.00515/full.

16. Treff G, Winkert K, Sareban M, Steinacker JM, Sperlich B. The PolarizationIndex: a simple calculation to distinguish polarized from non-polarized training intensity distributions. Front Physiol. 2019. https://doi.org/10. 3389/fphys.2019.00707/full.

17. Matzka M, Zinner C, Kunz P, Holmberg H-C, Sperlich B. Comparison of physiological parameters during on-water and ergometer kayaking and their relationship to performance in sprint kayak competitions. Int J Sports Physiol Perform. 2021;16:958-64.

18. Winchcombe CE, Binnie MJ, Doyle MM, Hogan C, Peeling P. Development of an on-water graded exercise test for flat-water sprint kayak athletes. Int J Sports Physiol Perform. 2019;1-21.

19. Carrasco Páez L, Martínez Díaz IC, del Hoyo Lora M, Sañudo Corrales FB, Ochiana N. Ergometric testing for top-level kayakers: validity and reliability of a discontinuous graded exercise test. Kinesiol Slov. 2010;16:16-20.

20. Zinner C, Schäfer Olstad D, Sperlich B. Mesocycles with different training intensity distribution in recreational runners. Med Sci Sports Exerc. 2018;50:1641-8.

21. Borg G. Perceived exertion as an indicator of somatic stress. Scand J Rehabil Med. 1970;2:92-8.

22. Sanders D, Myers T, Akubat I. Training-intensity distribution in road cyclists: objective versus subjective measures. Int J Sports Physiol Perform. 2017;12:1232-7.

23. Buchheit M, Laursen PB. High-intensity interval training, solutions to the programming puzzle: part l: cardiopulmonary emphasis. Sports Med Auckl NZ. 2013:43:313-38

24. van Someren KA, Howatson G. Prediction of flatwater kayaking performance. Int J Sports Physiol Perform. 2008;3:207-18. 
25. Hogan C, Binnie MJ, Doyle M, Lester L, Peeling P. Comparison of training monitoring and prescription methods in sprint kayaking. Int J Sports Physiol Perform. 2019;15:654-62.

26. Hogan C, Binnie MJ, Doyle M, Lester L, Peeling P. Heart rate and stroke rate misrepresent supramaximal sprint kayak training as quantified by power. Eur J Sport Sci. 2021;21:656-65.

27. Pérez-Landaluce J, Rodríguez-Alonso M, Fernandez-Garcia B, BustilloFernandez $\mathrm{E}$, Terrados N. Importance of wash riding in kayaking training and competition. Med Sci Sports Exerc. 1998;30:1721-4.

28. Esteve-Lanao J, San Juan AF, Earnest CP, Foster C, Lucia A. How do endurance runners actually train? Relationship with competition performance. Med Sci Sports Exerc. 2005;37:496-504.

29. Lucía A, Hoyos J, Pardo J, Chicharro JL. Metabolic and neuromuscular adaptations to endurance training in professional cyclists: a longitudinal study. Jpn J Physiol. 2000;50:381-8.

30. Plews DJ, Laursen PB, Kilding AE, Buchheit M. Heart-rate variability and training-intensity distribution in elite rowers. Int J Sports Physiol Perform. 2014;9:1026-32.

31. Schumacher YO, Mueller P. The 4000-m team pursuit cycling world record: theoretical and practical aspects. Med Sci Sports Exerc. 2002;34:1029-36.

32. Sylta $\varnothing$, Tønnessen E, Seiler S. From heart-rate data to training quantification: a comparison of 3 methods of training-intensity analysis. Int J Sports Physiol Perform. 2014;9:100-7.

33. Zapico AG, Calderón FJ, Benito PJ, González CB, Parisi A, Pigozzi F, DiSalvo $\checkmark$. Evolution of physiological and haematological parameters with training load in elite male road cyclists: a longitudinal study. J Sports Med Phys Fitness. 2007;47:191-6.

34. Sandbakk $\varnothing$, Holmberg H-C, Leirdal S, Ettema G. The physiology of worldclass sprint skiers. Scand J Med Sci Sports. 2011;21:e9-16.

35. Kenneally M, Casado A, Gomez-Ezeiza J, Santos-Concejero J. Training characteristics of a World Championship 5000-m finalist and multiple continental record holder over the year leading to a World Championship final. Int J Sports Physiol Perform. 2021;1-5.

36. Plisk S. Training principles and program design. Strategies Routledge. 2005;18:16-21.

37. Kiely J. Periodization theory: confronting an Inconvenient Truth. Sports Med. 2018;48:753-64.

38. Sperlich B, Holmberg $\mathrm{H}-\mathrm{C}$. The responses of elite athletes to exercise: an all-day, 24-h integrative view is required! Front Physiol. 2017;8:564.

\section{Publisher's Note}

Springer Nature remains neutral with regard to jurisdictional claims in published maps and institutional affiliations.

\section{Submit your manuscript to a SpringerOpen ${ }^{\circ}$ journal and benefit from:}

- Convenient online submission

- Rigorous peer review

- Open access: articles freely available online

- High visibility within the field

- Retaining the copyright to your article 